\title{
La demora diagnóstica en el cáncer colorrectal en función del medio de procedencia
}

\author{
Elena Pereiro Sánchez ${ }^{a}$, Gabriel J. Díaz Grávalos ${ }^{\mathrm{b}}$, Carmen M. Gándara Quintas ${ }^{\mathrm{c}}$, \\ María José Varela Estévez ${ }^{c}$, Alberto J. del Álamo Alonso ${ }^{a}$, Inmaculada Casado Górriz ${ }^{d}$
}

${ }^{a}$ Centro de Salud Nóvoa Santos (Orense). SERGAS.

${ }^{\mathrm{b}}$ Centro de Salud Cea (Orense). SERGAS.

${ }^{\circ}$ Centro de Salud Valle Inclán (Orense). SERGAS.

${ }^{d}$ Centro de Salud Allariz

(Orense). SERGAS.

Correspondencia: Gabriel J. Díaz Grávalos. C/ Feria, 9. C.P. 32130 - Cea (Orense). Teléfono: 619125 841. Correo electrónico: gabriel.diaz.gravalos@sergas.es

Un resumen de este estudio fue presentado como comunicación oral en las XV Xornadas Galegas de Medicina Familiar e Comunitaria (La Coruña, 12-13 de noviembre de 2010).

Recibido el 7 de marzo de 2012.

Aceptado para su publicación el 9 de septiembre de 2012.

\section{RESUMEN}

Objetivo: Determinar la existencia de diferencias en la demora diagnóstica del cáncer colorrectal atribuible al proveedor en función del medio de procedencia (rural/urbano) y valorar la repercusión que esa demora pudiera producir sobre la extensión tumoral medida mediante la estadificación.

Diseño del estudio: Estudio observacional transversal.

Emplazamiento: Atención sanitaria en la provincia de Orense.

Participantes: Todos los pacientes diagnosticados de cáncer colorrectal registrados en el Complejo Hospitalario de Orense en los años 2006 y 2007.

Mediciones principales: Se determinó: sexo, edad al diagnóstico, medio de procedencia (rural/urbano), fecha del primer contacto del paciente con el sistema sanitario (médico de familia, urgencias, otros), fecha de estadificación, grado de extensión al diagnóstico y demora diagnóstica (fecha de estadificación menos fecha de primer contacto del paciente).

Resultados: Fueron incluidos 549 casos, con una media de edad de 72,6 años (DE 11,2) y $64,5 \%$ de procedencia rural. No se constataron diferencias significativas en la estadificación entre ambos medios. La media de la demora diagnóstica era de 87,2 días (DE 119,7), siendo significativamente mayor en el medio rural $(95,5$ días [DE 135,5$])$ frente al urbano $(71,5$ días [DE $79,7])$. La regresión lineal mostró que una mayor demora se asociaba a la procedencia rural. Conclusiones: En el cáncer colorrectal, la procedencia rural supone una mayor demora diagnóstica atribuible al proveedor respecto al medio urbano, aunque no se acompaña de una estadificación más avanzada.

Palabras clave: Neoplasias colorrectales, Diagnóstico tardío, Estadificación de neoplasias, Población rural, Población urbana, Desigualdades en la salud.

\section{ABSTRACT}

Delayed diagnosis in colorectal cancer based on background

Objective: To determine the existence of differences in the delayed diagnosis of colorectal cancer attributable to the provider based on background (rural/urban) and to assess the repercussion that the delay may produce on the extension of the tumour by staging.

Design of the study: Cross-sectional observational study

Location: Health care service in the province of Orense.

Participants: All patients diagnosed with colorectal cancer registered at the Orense Hospital Complex in the years 2006 y 2007.

Main measurements. The following were determined: sex and age at time of diagnosis, background (rural/urban), date of the patient's first contact with the health system (family practitioner, emergencies, other), date of staging, degree of extension at time of diagnosis and delay in diagnosis (date of staging minus date of patient's first contact)

Results: 549 cases were included, with an average age of 72,6 years (DE 11,2) and $64,5 \%$ from a rural background. No significant differences in staging were found between the two areas. The mean delay in diagnosis was 87,2 days (DE 119,7), with a much higher incidence in the rural area $(95,5$ days [DE 135,5$])$ than in the urban area $(71,5$ days [DE 79,7$])$. The lineal regression showed that a longer delay was associated with the rural area.

Conclusions: In colorectal cancer, a rural background implies a greater delay in diagnosis attributable to the provider than in an urban area, although it is not accompanied by (associated with) a more advanced staging.

Key words: Colorectal neoplasms, Delayed diagnosis, Neoplasm staging, Rural population, Urban population, Health inequalities. 


\section{INTRODUCCIÓN}

En los países occidentales, el cáncer colorrectal (CCR) ocupa el segundo lugar en incidencia y mortalidad por cáncer, tanto en varones como en mujeres, y el primer lugar si se consideran ambos sexos conjuntamente, siendo la situación en España asimilable a la descrita ${ }^{1}$.

Existen algunas circunstancias que pueden alterar el resultado del tratamiento de esta enfermedad, aparte de las características propias del proceso y de la mejora de nuestras capacidades en su manejo. Puesto que el principal factor pronóstico del CCR es el estadio en el momento del diagnóstico ${ }^{2}$, una de esas circunstancias, al menos a nivel individual, es la demora que pueda afectar los procesos diagnóstico y terapéutico.

Hansen et $\mathrm{al}^{3}$ clasifican la demora en tres fases: atribuibles al paciente (tiempo que transcurre desde que presenta síntomas hasta que consulta al médico por primera vez por este motivo), al médico de Atención Primaria (tiempo que transcurre desde el primer contacto con el mismo hasta su remisión al segundo nivel asistencial) y al sistema (desde su remisión al segundo nivel hasta el diagnóstico definitivo). La suma de las demoras atribuibles al médico de Atención Primaria y al sistema se denomina también demora del proveedor.

Existen pruebas de que los pacientes que viven en medios más desfavorecidos socioculturalmente, entre ellos el rural, presentan desventaja frente al paciente de medio urbano en los resultados sanitarios de diversas enfermedades ${ }^{4,5}$, incluyendo el cáncer; tienen un acceso más limitado al sistema sanitario (mayor distancia, déficit de medios de transporte) y menor disponibilidad de información general y de programas educativos, constituyendo un ejemplo de las denominadas desigualdades sociales en salud ${ }^{6}$.

Teniendo en cuenta la presencia de datos que prueban la existencia en nuestro medio de diferencias en la demora diagnóstica en otros tipos de tumores ${ }^{7}$, aparentemente mediadas por factores externos a la enfermedad, se ha planteado el presente estudio con el objetivo de determinar si existen diferencias en la demora diagnóstica atribuible al proveedor en función del medio de procedencia (rural/urbano) y valorar la repercusión que esa demora pudiera producir sobre la extensión tumoral del cáncer colorrectal medida mediante la estadificación.

\section{SUJETOS Y MÉTODOS}

Se realizó un estudio transversal de base poblacional. Fueron incluidos todos los pacientes diagnosticados de cáncer colorrectal registrados en los años 2006 y 2007 en el Complejo Hospitalario de Orense, que presta asistencia a una población de 335.000 habitantes. Se excluyeron los pacientes en los que se constató la ausencia en su historia clínica de los datos necesarios para realizar los cálculos precisos para el estudio. El estudio se diseñó para comparar la variable "demora diagnóstica" (cuantitativa) en dos grupos definidos por la variable "procedencia" (cualitativa). A partir de una prueba piloto realizada con 20 casos escogidos de forma aleatoria se determinó la media en días y desviación estándar de la variable "demora diagnóstica" $(104,3 ; \mathrm{DE}=136,3)$ con la finalidad de utilizarla para el cálculo del tamaño de muestra necesario. Considerando una precisión de 30 días y una confianza del $95 \%$ se determinó un tamaño de muestra mínimo de 316 individuos.

Se registraron las siguientes variables: Sexo, edad en años en el momento del diagnóstico, medio de procedencia (rural / urbano) determinado según los criterios recomendados por la OCDE (rural: $<150$ habitantes $/ \mathrm{km}^{2}$ ), antecedentes familiares de cáncer colorrectal, fecha de diagnóstico de sospecha, considerando así la fecha del primer contacto del paciente con el sistema sanitario (médico de familia, servicio de urgencias, otros) a causa de la enfermedad, fecha de diagnóstico de extensión (se consideró así la fecha de estadificación), fecha de realización de la colonoscopia, grado de extensión del tumor al diagnóstico o estadificación (medida mediante la clasificación del American Joint Committee on Cancer $\left.[A J C C]^{8}\right)$, y demora diagnóstica atribuible al proveedor (DD), que se calculó a partir de la diferencia entre la fecha de diagnóstico definitivo, considerándose así la fecha de estadificación, y la fecha del diagnóstico de sospecha, considerándose así la fecha del primer contacto del paciente con el sistema sanitario a causa de la enfermedad.

Para facilitar los cálculos se transformó la variable cualitativa "grado de extensión" en una variable cuantitativa en la que a mayor valor, mayor extensión, dando un valor progresivo a cada uno de los estadios de la clasificación del AJCC (tabla 1).

Los datos fueron recogidos a lo largo de 2010, proporcionados por el Servicio de Documentación Clínica del Complejo Hospitalario de Orense, a partir de las historias clínicas. 


\begin{tabular}{|c|c|c|c|c|}
\hline Estadios AJCC & $\mathbf{T}$ & $\mathbf{N}$ & M & $\begin{array}{l}\text { Grado de } \\
\text { extensión }\end{array}$ \\
\hline Estadio 0 & TIS & No & Mo & 1 \\
\hline \multirow[t]{2}{*}{ Estadio I } & $\mathrm{T} 1$ & No & Mo & 2 \\
\hline & T2 & No & Mo & 2 \\
\hline Estadio IIA & T3 & No & Mo & 3 \\
\hline Estadio IIB & T4 & No & Mo & 4 \\
\hline \multirow[t]{2}{*}{ Estadio IIIA } & $\mathrm{T} 1$ & $\mathrm{~N} 1$ & Mo & 5 \\
\hline & T2 & $\mathrm{N} 1$ & Mo & 5 \\
\hline \multirow[t]{2}{*}{ Estadio IIIB } & T3 & $\mathrm{N} 1$ & Mo & 6 \\
\hline & $\mathrm{T} 4$ & N1 & MO & 6 \\
\hline Estadio IIIC & Cualquier $\mathrm{T}$ & $\mathrm{N} 2$ & Mo & 7 \\
\hline Estadio IV & Cualquier $T$ & Cualquier N & M1 & 8 \\
\hline
\end{tabular}

AJCC: American Joint Committee on Cancer $^{8}$

Tabla 1. Equivalencia numérica utilizada en la transformación de la variable cuantitativa "grado de extensión" a partir de la estadificación del cáncer colorrectal según el American Joint Committee on Cancer y la clasificación TNM.

El análisis de los datos se realizó con ayuda del programa estadístico SPSS versión 15.0 (SPSS Inc.). Las variables cuantitativas fueron descritas como media y desviación estándar y las cualitativas como porcentajes. Se determinaron los intervalos de confianza del 95\%.

Se utilizaron en el análisis bivariable las pruebas estadísticas necesarias según las variables implicadas y previa comprobación del cumplimiento de sus condiciones. Así, para la comparación de variables cualitativas dicotómicas se empleó la prueba de $\mathrm{X}^{2}$. La comparación de medias se realizó mediante la t de Student. Se determinó la relación entre variables cuantitativas mediante la correlación de Spearman.

El análisis multivariable se realizó mediante regresión lineal, considerando como variable dependiente la DD e incluyéndose en el modelo edad, sexo y medio de procedencia. Se utilizó un método de selección de variables por pasos hacia atrás.

El grado de significación estadística que se consideró fue un valor de $\mathrm{p}<0,05$.

\section{RESULTADOS}

Fueron incluidos 549 casos, siendo varones 319 $(58,1 \%)$ y perteneciendo al medio rural 354 casos $(64,5 \%)$. La media de la edad fue de 72,6 años (DE 11,2) (IC95\% 71,7-73,6), sin diferencias en función del medio de procedencia $(t=1,4 ; p=$ $0,13)$. Fueron excluidos 9 casos por falta de alguno de los datos necesarios para realizar los cálculos. La tabla 2 muestra las principales características sociodemográficas y clínicas recogidas.

La media de la demora en la realización de la colonoscopia para el medio rural era de 1,7 $(4,4)$ meses y en el urbano de $1,1(2,5)$ meses, sin diferencias significativas. No se demostró diferencia significativa entre la edad al diagnóstico 


\begin{tabular}{|c|c|c|}
\hline Variables & Rural (354) & Urbano (186) \\
\hline \\
\hline \multicolumn{3}{|l|}{ Sexo } \\
\hline Varón & $214(60,5 \%)$ & $105(56,5 \%)$ \\
\hline Mujer & $140(39,5 \%)$ & $81(43,5 \%)$ \\
\hline \multicolumn{3}{|l|}{ Antecedentes familiares } \\
\hline Sí & 72 & 56 \\
\hline No & 95 & 34 \\
\hline \multicolumn{3}{|l|}{ Estadificación } \\
\hline 0 & 1 & 1 \\
\hline I & 52 & 23 \\
\hline IIA & 78 & 43 \\
\hline IIB & 18 & 7 \\
\hline IIIA & 3 & 2 \\
\hline IIIB & 63 & 29 \\
\hline IIIC & 34 & 21 \\
\hline IV & 96 & 54 \\
\hline
\end{tabular}

Tabla 2. Características de los pacientes incluidos en el estudio.

\begin{tabular}{|l|c|c|c|}
\hline \multicolumn{1}{|c|}{ Variable } & Coeficiente Beta & IC95\% & p \\
\hline Medio (rural) & 24,8 & $3,5-46,1$ & 0,02 \\
\hline Edad & $-0,29$ & $-1,2-0,6$ & 0,5 \\
\hline Sexo & $-17,8$ & $-38,4-2,8$ & 0,09 \\
\hline
\end{tabular}

Tabla 3. Resultado de la regresión lineal considerando como variable dependiente la demora diagnóstica.

y la demora para la colonoscopia. Para el medio rural se obtuvo una media de estadificación de 5,25 y para el medio urbano de 5,37, valores que se corresponderían con los estadios IIIA y IIIB del American Joint Committee on Cancer, sin diferencias significativas $(t=0,6 ; p=0,6)$.

La media de la DD era de $87,2(119,7)$ días (IC95\% $77,1-97,4)$, con una mediana de 54,0 días. El $29,9 \%$ de los casos presentaban una DD mayor de 3 meses, sin demostrarse diferencias significativas en función del medio de procedencia en estos casos de mayor demora $\left(X^{2}=3,2 ; p=0,08\right)$.

La DD era mayor en el medio rural (media 95,5 días [135,5]; mediana 60,0 días) frente a la del medio urbano (media 71,5 días [79,7]; mediana 46,5 días) (diferencia de medias: 24,1 días; $p=0,01$; IC95\%
2,8 - 45,4). La regresión lineal mostró que una mayor demora diagnóstica estaba asociada a la procedencia del medio rural. La tabla 3 muestra los resultados obtenidos en el análisis.

\section{DISCUSIÓN}

Los resultados de este estudio muestran que la procedencia del medio rural supone una mayor demora diagnóstica atribuible al proveedor en el CCR respecto a la procedencia del medio urbano, aunque esta mayor demora no se acompaña de una estadificación más avanzada.

Las diferencias en resultados sanitarios entre medio rural y urbano es un tópico estudiado en diferentes áreas de la medicina y con resultados contradictorios. En general, se acepta que la 
pertenencia al medio rural dificulta la consecución de determinados objetivos en salud ${ }^{9}$. Así, y ciñéndose al manejo de la enfermedad neoplásica, los pacientes rurales presentan desventaja en el estudio diagnóstico, el seguimiento posterior al tratamiento o la participación en ensayos clínicos $^{10,11}$. Existe incluso, por parte de los pacientes, la creencia de que su pertenencia al medio rural se traducirá en peores resultados en el tratamiento del cáncer ${ }^{12}$. En el caso concreto del CCR hay evidencias que muestran una mayor mortalidad asociada a la residencia en medio rural ${ }^{13}$, e incluso después de ajustar por privación, el riesgo de mortalidad fue significativamente más alto en zonas rurales.

Los escasos estudios que han analizado las diferencias en esta área han encontrado resultados similares al presente. Así, Robinson et $\mathrm{al}^{14}$ en Israel o Bain et $\mathrm{al}^{15}$ en Escocia encontraron mayor demora entre los pacientes que vivían en el área rural frente a la urbana, con un grado de evidencia fuerte según la revisión sistemática de Mitchell ${ }^{16}$. A la misma conclusión llegaron Fourne ${ }^{17}$ o Launoy ${ }^{18}$, aunque en este último caso sólo se encontraron diferencias significativas en mujeres. Parece que incluso los médicos que trabajan en la zona rural aceptan que la mayor distancia a los centros de referencia influirá negativamente en los resultados ${ }^{19}$.

La duración global de la demora en este estudio es similar a las recogidas en la literatura ${ }^{16}$, independientemente del sistema sanitario que se considere, oscilando desde los 41 días de Wattacherill ${ }^{20}$ en EE.UU, hasta los $69{\text { de } \text { Barret }^{21} \text { en }}^{2}$ Reino Unido, o los 59 encontrados por Van Hout en Países Bajos ${ }^{22}$.

Aceptado que las diferencias en la supervivencia se ven afectadas por el estadio en el momento del diagnóstico ${ }^{3}$, existen datos contradictorios en cuanto a la relación entre mayor demora y estadificación más avanzada. Hay estudios que demuestran incluso que a una mayor demora en el diagnóstico de CCR se le asocia un estadio menos avanzado ${ }^{23}$.

Algunos autores obtienen resultados asimilables a los de este estudio, sin apreciar diferencias en la estadificación en función del medio de procedencia ${ }^{24}$, ni de la demora diagnóstica ${ }^{25}$. En otros casos se encuentra que vivir en áreas rurales o a mayor distancia de hospitales que prestan asistencia a pacientes oncológicos se ha asociado con estadios más avanzados en el momento del diagnóstico o menor probabilidad de presentar tumores in situ ${ }^{26-30}$, aunque también aquí existen resultados contrapuestos, y así Koka et $a^{31}$ encontró que la distancia a un centro hospitalario terciario no parecía una barrera para el diagnóstico temprano de CCR.

Para explicar estas diferencias se han apuntado diversas hipótesis, entre ellas la asociación entre situación socioeconómica y medio rural. Pudiera ser que la situación socioeconómica fuera un determinante más importante de una estadificación temprana que la residencia urbana o rural. Así, en Florida se encontró que la mala situación socioeconómica se asoció a enfermedad en estadio avanzado, pero no el hecho de la residencia rural ${ }^{32}$. Igualmente, en el Reino Unido la mala situación económica se asoció con una peor supervivencia ${ }^{33}$. Este es un punto que debería ser investigado a través de la utilización de un índice de privación adecuado a nuestro medio.

A la hora de valorar los resultados de este estudio es necesario considerar las limitaciones relativas al sistema de recogida de datos, realizado a partir de historias clínicas, y los problemas inherentes a la posible falta de datos o errores en las mismas.

Dadas las características de la patología considerada, la historia clínica suele contener con bastante precisión la fecha del primer contacto del paciente con el sistema sanitario, así como la fecha de estadificación definitiva.

Por otra parte, el estudio incluye todos los casos de CCR atendidos por el Servicio sanitario público de la provincia en el periodo considerado, por lo que puede considerarse que representa ampliamente la totalidad de los casos acaecidos en ese periodo en el territorio.

Una consideración importante en cualquier estudio sobre la ruralidad es el propio concepto o, si se prefiere, la diferencia entre rural y urbano. Puesto que la ruralidad se presenta habitualmente como una característica dicotómica, esta categorización puede enmascarar diferencias importantes y producir una mala clasificación en una cierta proporción de la población. En el presente estudio se ha recurrido a un criterio internacionalmente aceptado con el propósito de evitar en lo posible este hecho.

\section{BIBLIOGRAFÍA}

1. Ferlay J, Shin H, Bray F, Forman D, Mathers C, Parkin DM. Estimates of worldwide burden of cancer in 2008: GLOBOCAN 2008. Int J Cancer. 2010;127(12):2893-917.

2. Gatta G, Capocaccia R, Sant M, Bell CMJ, Coebergh JWW, Damhuis RAM et al. Understanding variations in survival for colorectal cancer in Europe: a EUROCARE high resolution 
study. Gut. 2000;47(4):533-8.

3. Hansen RP, Olesen F, Sørensen HT, Sokolowsky I, Søndergaard J. Socioeconomic patient characteristics predict delay in cancer diagnosis: a Danish cohort study. BMC Health Serv Res. 2008;8:49.

4. Downing A, Prakash K, Gilthorpe MS, Mikeljevic JS, Forman D. Socioeconomic background in relation to stage at diagnosis, treatment and survival in women with breast cancer. Br J Cancer. 2007;96(5):836-40.

5. Turón JM, Librero J, Díaz GJ, García JJ, Beltral C, Abal F on behalf of the REDIMER study group (semFYC). Auditing secondary prevention of ischemic heart disease in rural areas of Spain: an opportunity for improvement. Eur J Gen Pract. 2006;1(4)2:156-62.

6. Solar O, Irwin A. A conceptual framework for action on the social determinants of health. Social determinants of health discussion paper 2 (policy and practice). Geneva: World Health Organization; 2010.

7. López González A. La ruralidad como determinante de la demora diagnóstica en el cáncer de mama. Cad Aten Primaria. 2009;16(3):193-6.

8. Green FL, Page DL, Fleming ID, Fritz A, Balch CM, Haller DG et al, eds. American Joint Committee on Cancer: AJCC Cancer Staging Manual. 6th ed. New York: Springer; 2002.

9. Auchincloss A, Hadden W. The health effects of ruralurban residence and concentrated poverty. J Rural Health. 2002;18(2):319-36.

10. Elliott TE, Elliott BA, Renier CM, Haller IV. Rural-urban differences in cancer care: results from the Lake Superior Rural Cancer Care Project. Minn Med. 2004;87(9):44-50.

11. Kinney AY, Harrell J, Slattery M, Martin C, Sandler RS. Ruralurban differences in colon cancer risk in blacks and whites: the North Carolina Colon Cancer Study. J Rural Health. 2006;22(2):124-30.

12. Bain NSC, Campbell NC. Treating patients with colorectal cancer in rural and urban areas: a qualitative study of the patient's perspective. Fam Pract. 2000;17(6):475-9.

13. Singh GK, Williams SD, Siahpush M, Mulhollen A. Socioeconomic, rural-urban, and racial inequalities in US cancer mortality: Part I - All cancers and lung cancer and Part II - Colorectal, prostate, breast, and cervical cancer. J Cancer Epidemiol. 2011;2011:107497.

14. Robinson E, Mohilever J, Zidan J, Sapir D. Colorectal cancer: incidence, delay in diagnosis and stage of disease. Eur $\mathrm{J}$ Cancer Clin Oncol. 1986;22(2):157-61.

15. Bain NSC, Campbell NC, Ritchie LD, Cassidy J. Striking the right balance in colorectal cancer - a qualitative study of rural and urban patients. Fam Pract. 2002;19(4):369-74.

16. Mitchell E, Macdonald S, Campbell NC, Weller D, Macleod U. Influences on pre-hospital delay in the diagnosis of colorectal cancer: a systematic review. Br J Cancer. 2008;98(1):60-70.

17. Fournel I, Cottet V, Binquet C, Jooste V, Faivre J, Bouvier AM et al. Rural-urban inequalities in detection rates of colorectal tumours in the population. Dig Liver Dis. 2012;44(2):172-7.

18. Launoy G, Le Coutour X, Gignoux M, Pottier D, Dugleux G. Influence of rural environment on diagnosis, treatment, and prognosis of colorectal cancer. J Epidemiol Community Health. 1992;46(4):365-7.

19. Jiwa M, Halkett G, Aoun S, Arnet $H$, Smith M, Pilkington $M$ et al. Factors influencing the speed of cancer diagnosis in rural Western Australia: a General Practice perspective. BMC Fam Pract. 2007;8:27.

20. Wattacheril J, Kramer JR, Richardson P, Havemann BD, Green LK, Le A, et al. Lagtimes in diagnosis and treatment colorectal cancer: determinants and association with cancer stage and survival. Aliment Pharmacol Ther. 2008;28(9):1166-74.

21. Barrett J, Jiwa M, Rose P, Hamilton W. Pathways to the diagnosis of colorectal cancer: an observational study in three UK cities. Fam Pract. 2006;23(1):15-9.

22. Van Hout AM, de Wit NJ, Rutten FH, Peeters PH. Determinants of patient's and doctor's delay in diagnosis and treatment of colorectal cancer. Eur J Gastroenterol Hepatol. 2011;23(11):1056-63.

23. Roncoroni L, Pietra N, Violi V, Sarli L, Choua O, Peraccia A. Delay in the diagnosis and outcome of colorectal cancer: a prospective study. Eur J Surg Oncol. 1999;25(2):173-8. 24. Gómez-Domínguez E, Trapero-Marugán M, Del Pozo AJ, Cantero J, Gisbert JP, Maté J. Factores pronósticos en carcinoma colorrectal. Importancia de la demora diagnóstica. Rev Esp Enferm Dig. 2006;98(5):322-9.

25. Ramos M, Esteva M, Cabeza E, Llobera J, Ruiz A. Lack of association between diagnostic and therapeutic delay and stage of colorectal cancer. Eur J Cancer. 2008;44(4):510-21. 26. Campbell NC, Elliott AM, Sharp L, Ritchie LD, Cassidy J, Little J. Rural and urban differences in stage at diagnosis of colorectal and lung cancers. Br J Cancer. 2001;84(7):910-4.

27. Higginbotham JC, Moulder J, Currier M. Rural v. urban aspects of cancer: first-year data from the Mississippi Central Cancer Registry. Fam Community Health. 2001;24(2):1-9.

28. Hines RB, Markossian TW. Differences in late-stage diagnosis, treatment, and colorectal cancer-related death between rural and urban African Americans and whites in Georgia. J Rural Health. 2012;28(3):296-305.

29. Palmer RC, Schneider EC. Social disparities across the continuum of colorectal cancer: A systematic review. Cancer Causes Control. 2005;16(1):55-61.

30. Parsons MA, Askland KD. Cancer of the colorectum in Maine, 1995-1998: determinants of stage at diagnosis in a rural state. J Rural Health. 2007;23(1):25-32.

31. Koka VK, Potti A, Fraiman GN, Hanekom D, Hanley JF. An epidemiological study evaluating the relationship of distance from a tertiary care cancer center to early detection of colorectal carcinoma. Anticancer Res. 2002;22(4):2481-3.

32. Campbell RJ, Ferrante JM, Gonzalez EC, Roetzheim RG, Pal N, Herold A. Predictors of advanced stage colorectal cancer diagnosis: results of a population-based study. Cancer Detect Prev. 2001;25(5):430-8.

33. Lejeune C, Sassi F, Ellis L, Godward S, Mark V, Day M et al. Socio-economic disparities in access to treatment and their impact on colorectal cancer survival. Int $\mathrm{J}$ Epidemiol. 2010;39(3):710-7 